\title{
RESEARCH
}

Open Access

\section{Hippocampal magnetic resonance imaging in focal onset seizure with impaired awareness-descriptive study from tertiary care centre in southern part of India}

Aleena Elizabeth Andrews ${ }^{1}$, Naufal Perumpalath², Juvaina Puthiyakam² and Andrews Mekkattukunnel ${ }^{3,4^{*}}$

\begin{abstract}
Background: Temporal lobe epilepsy is the most common type of focal onset seizure. Focal onset seizure with impaired awareness, previously known as complex partial seizure (CPS), account for $18-40 \%$ of all seizure types. Hippocampal sclerosis (HS) is the most common cause of temporal lobe epilepsy, which produces focal onset seizure with impaired awareness. It may be detected in MRI visually, but bilateral abnormalities are better identified using volumetric analysis.

We aimed to compare hippocampal volume in patients with focal onset seizure with impaired awareness visually and quantitatively.
\end{abstract}

Methodology: This cross-sectional study includes clinically diagnosed cases of 56 focal onset seizure with impaired awareness undergoing MRI at a tertiary teaching hospital in the southern part of India for a duration of 18 months from February 2018 to August 2019.

Results: Out of 53 patients studied using 1.5 T MRI brain with seizure protocols, hippocampal atrophy was identified visually in 13 (24.5\%) on the right side, 9 (16.98\%) on the left side, and in 6 (11.32\%) bilaterally. However, with volumetry, hippocampal atrophy (not taking T2 signal change) was detected in 15 (28.30\%) on the right side, 10 (18.86\%) on the left side, and in 7 (13.20\%) bilaterally. Hippocampal volumes between ipsilateral and contralateral seizure focus were found to have no significant difference ( $p-0.84)$.

Conclusions: Though visual analysis is efficient in the diagnosis of pathology, MR volumetry may be used as an expert eye in cases of subtle volume loss.

Keywords: Focal onset seizure with impaired awareness, Complex partial seizures, Mesial temporal lobe epilepsy (MTLE), Hippocampal sclerosis (HS), Hippocampal volumetry

\footnotetext{
* Correspondence: anjulioness@gmail.com

${ }^{3}$ Professor of Medicine, Government Medical College Thrissur, Thrissur, Kerala

680002, India

${ }^{4}$ Mekkattukunnel House, Chakkoth lane, Poonkunnam, Thrissur, Kerala

680002 , India

Full list of author information is available at the end of the article
}

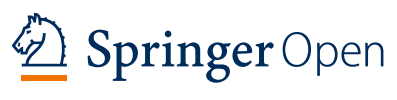

(c) The Author(s). 2021 Open Access This article is licensed under a Creative Commons Attribution 4.0 International License, which permits use, sharing, adaptation, distribution and reproduction in any medium or format, as long as you give appropriate credit to the original author(s) and the source, provide a link to the Creative Commons licence, and indicate if changes were made. The images or other third party material in this article are included in the article's Creative Commons licence, unless indicated otherwise in a credit line to the material. If material is not included in the article's Creative Commons licence and your intended use is not permitted by statutory regulation or exceeds the permitted use, you will need to obtain permission directly from the copyright holder. To view a copy of this licence, visit http://creativecommons.org/licenses/by/4.0/. 


\section{Background}

Epilepsy is characterized by tendency to have recurrent seizures [1] with prevalence of $0.5-1 \%$. Focal onset seizure with impaired awareness account for $18-40 \%$ of seizure types [2]. Focal onset seizure with impaired awareness can be of temporal and extra-temporal origin. The commonest form of temporal lobe epilepsy (TLE) is mesial temporal sclerosis where the pathology lies in hippocampus. TLE is responsible for two third cases of intractable epilepsy which is managed neurosurgically [3]. Most of the patients have good outcomes after surgery, and this depends on the evaluation by EEG and magnetic resonance imaging (MRI). Hippocampal sclerosis (HS) can be detected by visual inspection in most cases; however, volumetry can assist visual inspection if $[3,4]$ volume loss is subtle or bilateral resulting in lack of asymmetry, when the head is tilted while positioning in gantry or centres lack an expert in epilepsy imaging.

Many MR imaging studies interpreted as normal were later found to have hippocampal atrophy (HA) at tertiary epilepsy program [4]. There is a strong association between hippocampal asymmetry, identified by quantitative MR imaging, and visual inspection of the volumetric MR studies by two neuroradiologists who are trained to detect HA (91-97\%) [4]. Thus, volumetric MR imaging can serve as an expert "eye".

The mean volume of hippocampus is significantly smaller as compared to western population as well as northern regions of India, suggesting demographic variation in the hippocampal volume [5]. Very few data exist regarding the role of qualitative and quantitative hippocampal magnetic resonance imaging assessment in complex partial seizures from southern parts of the country.

\section{Methods}

This cross-sectional study was conducted among all clinically diagnosed cases of focal onset seizure with impaired awareness, referred for MRI brain with seizure protocol in 2500-bedded tertiary care hospital from Southern India for a duration of 18 months from February 2018 to August 2019. The study included eligible cases diagnosed by senior neurologist using semiological and EEG criteria as per the International League Against Epilepsy (ILAE) 2017 guidelines [6]. The study was approved by the Institutional Review Board of the tertiary teaching hospital on 26 October 2017. As this imaging evaluation is a routine part of management of epilepsy, the written consent was waived by the ethics committee. The research involved no more than minimal risk to subjects and waiver will not adversely affect the rights and welfare of the subjects.

MR imaging was performed on $1.5 \mathrm{~T}$ MRI scanner (Wipro GE Healthcare, closed type with bore size of 60 $\mathrm{cm}$, maximum amplitude of $33 \mathrm{mT} / \mathrm{m}$, USA in origin);
T1W, T2W, diffusion-weighted, and GRE sequences were obtained in axial plane with $5 \mathrm{~mm}$ slice thickness and $30 \%$ interslice gap. For dedicated hippocampal study, 3D T1w FSPGR (fast spoiled gradient echo sequence; inversion recovery prep) oblique coronal images (TE 5.8, TR 12.9, FOV $180 \mathrm{~mm}$, slice thickness $3 \mathrm{~mm}$ without interslice gap, matrix $288 \times 128\{$ FE XPE \}, NEX -0.75, phase FOV 1, prep time $400 \mathrm{~ms}$, flip angle 20, bandwidth of 15.63), T2W FLAIR coronal oblique images (TE: 95, TR: 8000, FOV: $160 \mathrm{~mm}$, slice thickness: 3 $\mathrm{mm}$ ), and oblique coronal T2W images (TR: 3700, TE: 97, FOV: 160, slice thickness: $6 \mathrm{~mm}$ ) covering the whole brain were acquired. Oblique coronal plane was perpendicular to the long axis of the hippocampus.

Visually, hippocampus was assessed in T2W images for size and signal intensity. All MRIs were assessed for regional atrophy independently by 2 radiologists, blinded to all clinical details except age. The two radiologists had almost perfect agreement on evaluation of hippocampal atrophy visually $(\kappa=0.8373)$.

The volume of the hippocampus was obtained from oblique coronal 3D T1w FSGR sequences. Crosssectional areas of both the hippocampi were measured in these oblique coronal sections by tracing hippocampal boundary manually from the hippocampal head to the tail. On MRI, the anterior-most boundary of the hippocampal head area is taken when the CSF in the uncal recess of the temporal horn was visible and is considered as the most reliable boundary between the hippocampal head and the amygdala. If uncal recess was not visible, then the alveus was used. To standardize the measurement, first section of the anterior hippocampus was defined as the point where the uncal recess or alveus first appears. Posterior margin of hippocampal volumetric measurement was defined by MR image where the crus of fornix were seen in full profile. Lateral and medial borders were defined as CSF in the temporal horn of the lateral ventricle and CSF in the uncal/ambient cisterns, respectively. Inferior border was defined by grey-white matter junction between the subiculum and white matter of the parahippocampal gyrus. The volumes of both hippocampi were calculated by summing each of the cross-sectional volumes cross sectional area $\times$ (section thickness + interslice gap)\}. As per study conducted by Mohandas, Aravind Narayan and colleagues on normative data of hippocampal volumetry Indian population, a mean hippocampal volume was found to be $2.411 \mathrm{~cm}^{3}$ [5]. This was much smaller as compared to the data available from the western population.

Statistical analysis was performed using percentages and proportions for qualitative data (visual assessment of hippocampal volume). Quantitative data (volumetry and $\mathrm{T} 2$ relaxometry of hippocampus) are presented as mean with standard deviation. The statistical significance 
of differences in mean volumes between right and left sides were assessed using the ' $t$ ' test.

Values that are 2 SD below the mean of normal and left-right asymmetries at least $2 \mathrm{SD}$ above or below the mean of normal are taken as abnormal for individual patients.

All analysis was done using epi info 7 version software (CDC open software, in Atlanta, GA, USA) and Microsoft Excel. Pearson's correlation coefficient and test were used wherever indicated. $\mathrm{p}$ value $<0.05$ was considered to be significant.

Initially visual assessment of the sections of the brain was performed, in which those cases without space occupying lesions/perilesional oedema involving hippocampus $(n=53)$ were assessed visually and subjected to volumetry using specific sequences (oblique coronal T2 FLAIR, oblique coronal three-dimensional T1w FSPGR).

\section{Results}

There were 56 patients in the study with a mean age of 26.44 with SD 14.78 years (see Fig. 1).

By the Shapiro-Wilk test of normality, it was found that the data differed significantly from normal distribution (test statistic 0.856; df 56; p value is 0.000). Age, gender, and febrile seizure distribution is depicted in Table 1.

Fifty-six patients with TLE were further divided into right TLE and left TLE groups based on the side of EEG localization. Six patients $(10.71 \%)$ whose seizure foci were either outside the temporal lobe or had a multifocal origin or could not be localized were grouped as extratemporal/unclassified (ET/UC).

Out of 56 patients evaluated with MRI, 13 patients (23.21\%) had mesial temporal sclerosis, seven had tumours, one had non-specific white matter T2 hyperintensity in the extratemporal location, 12 had hippocampal atrophy without signal alteration,1 had T2 hyperintensity of the hippocampus without volume loss, and $22(39.28 \%)$ had no obvious abnormalities in imaging with $1.5 \mathrm{~T}$ MRI. Three cases with perilesional oedema due to tumour extending into the hippocampus were excluded from analysis (see Fig. 1).

The major tumours detected were glioblastoma multiforme (n 2), dysembryoplastic neuroectodermal tumour (n 3), ganglioglioma (n 1), and glioma (n 1).

Using visual assessment, atrophy with T2 hyperintensity of the hippocampus was seen in $9(16.98 \%)$ on the right side, 5 on the left side (9.4\%), and bilaterally in 1 $(1.9 \%)$ only (Table 2 ).

Hippocampal atrophy with T2 hyperintensity suggestive of sclerosis (see Fig. 2) was seen in 9 (16.98\%) on the right side; however, there was a single case of T2 hyperintensity without atrophy.

In our study out of 53 cases, 13 cases (24.5\%) showed atrophy of mammillary bodies/fornix with temporal horn dilation ipsilateral to the side of the hippocampal atrophy. In the cases where there was bilateral hippocampal atrophy, it was seen that there was atrophy of either mammillary body or fornix and temporal horn dilation visually in both sides (see Fig. 3).

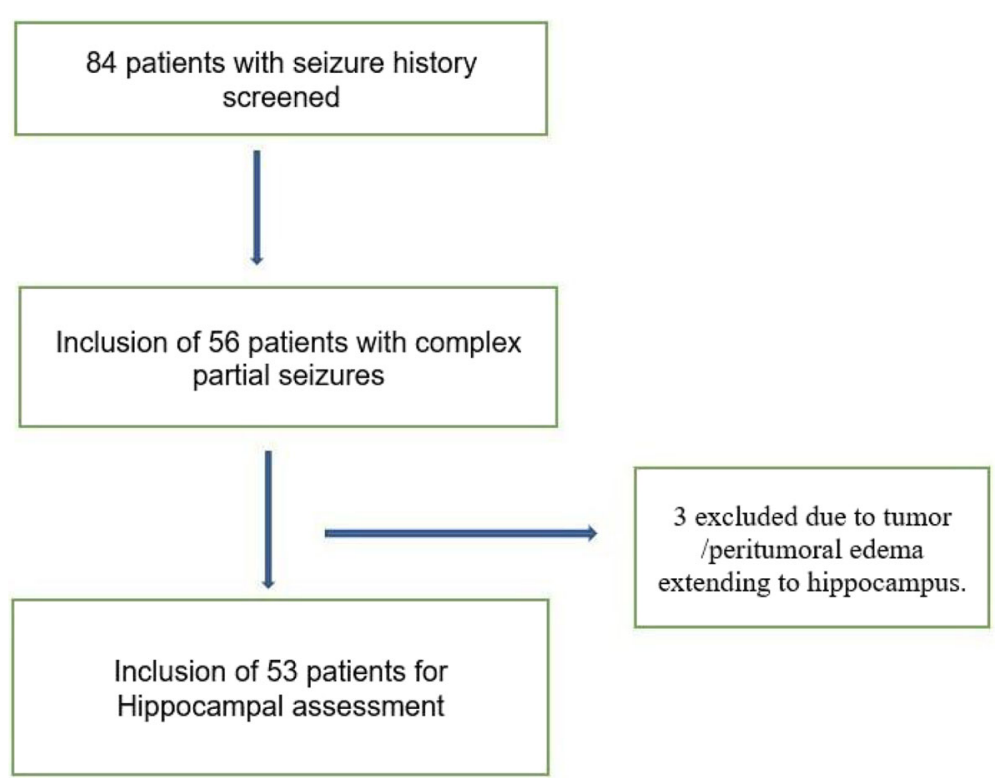

Fig. 1 Strobe diagram showing the methodology of the section of the cases for hippocampal assessment in patients presenting with focal onset seizure with impaired awareness 
Table 1 Age, gender, and presence of febrile seizures in patients with complex partial seizure (focal onset seizures with impaired awareness)

\begin{tabular}{llll}
\hline Gender & Number & Median Age with SD & Febrile Seizure \\
\hline Male & $30(53.57 \%)$ & $22(15.99)$ & $13(43.33 \%)$ \\
Female & $26(46.43 \%)$ & $19.5(13.40)$ & $6(23.36 \%)$ \\
\hline
\end{tabular}

To label hippocampal atrophy, a cut off value of $2.4 \mathrm{cc}$ was taken [4].

Among the 53 cases, 15 (28.30\%) had right-sided atrophy quantitatively (not taking T2 hyperintensity into consideration), 10 (18.87\%) had left sided atrophy, and 7 (13.20\%) had bilateral atrophy.

Combining hippocampal atrophy with signal alteration in T2, 7 had right hippocampal sclerosis, 4 had left hippocampal sclerosis, and 2 had bilateral hippocampal sclerosis (see Fig. 4).

Comparisons of right and left hippocampal volumes on ipsilateral and contralateral to seizure focus revealed no significant difference $(\mathrm{n}=53$, mean volume right 2.54, left $2.56 \mathrm{p}-0.80$ ).

Comparing the $\mathrm{T} 2$ relaxometry of the hippocampus in presumed normal cases and those with hippocampal sclerosis on either side showed a statistically significant difference (Table 3).

Comparisons of right and left hippocampal T2 relaxometry values ipsilateral and contralateral to seizure focus were analysed and were found to have no statistically significant difference $(n=53$, mean 81.44 on the right, 86 on the left and p-0.86).

\section{Discussion}

As per estimate, $1 \%$ of the world population suffers from epilepsy, of which the most common adult type is focal onset seizure with impaired awareness (previously known as complex partial seizure) [7]. Williamson PD, French JA, Thadani VM, Kim JH, and colleagues noted that more than $80 \%$ cause of TLE is mesial temporal lobe sclerosis (MTS) [8]. But in our study, 23.21\% showed features suggestive of MTS, seven had tumours, one had non-specific white matter T2 hyperintensity in extratemporal location, 12 had hippocampal atrophy

Table 2 Hippocampal atrophy and T2 hyperintensity distribution in CPS (focal onset seizures with impaired awareness)

\begin{tabular}{llll}
\hline & Right & Left & Bilateral \\
\hline Atrophy & $13(24.53 \%)$ & $9(16.98 \%)$ & $6(11.32 \%)$ \\
T2 hyperintensity & $10(18.87 \%)$ & $6(11.32 \%)$ & $1(1.9 \%)$ \\
Atrophy with T2 hyperintensity & $9(16.98 \%)$ & $5(9.4 \%)$ & $1(1.9 \%)$ \\
T2 hyperintensity without atrophy & $1(1.9 \%)$ & 0 & 0 \\
\hline
\end{tabular}

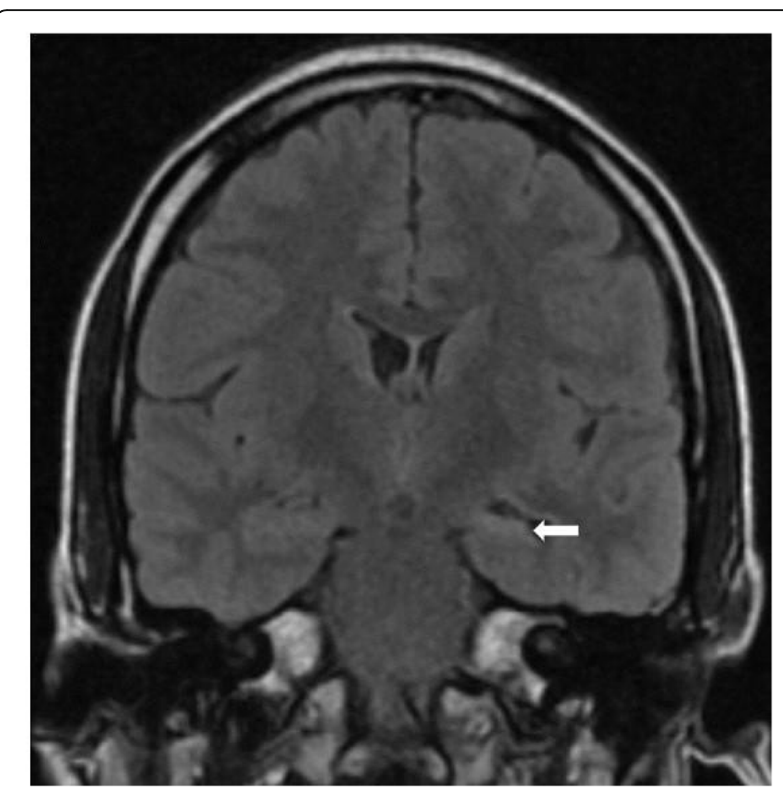

Fig. 2 Coronal T2 fluid-attenuated inversion recovery sequence MRI of the brain showing left hippocampal sclerosis (white arrow)

without signal alteration, and 1 had T2 hyperintensity of hippocampus without volume loss.

The major tumours detected in our study were glioblastoma multiforme (n 2), dysembryoplastic neuroectodermal tumour (n 3), ganglioglioma (n 1), and glioma (n 1 ). As per study by Brooks and colleagues, tumours were detected in $22 \%$ subjects [9].

In our study, 22 subjects (39.28\%) had no obvious abnormalities in imaging with $1.5 \mathrm{~T}$ MRI. As per Muhlhofer W, Tan Y-L, Mueller SG, and Knowlton R, up to $30 \%$ of TLE cases have normal ("non lesional" or negative) magnetic resonance imaging (MRI) [10].

Magnetic resonance imaging (MRI) features of hippocampal sclerosis (HS) by visual analysis of MRI as described by Cendes and colleagues are hippocampal atrophy, increased T2/FLAIR signal, loss of internal structure, asymmetry of the horns of the lateral ventricles, atrophy of the anterior temporal lobe, and atrophy of the ipsilateral fornix and mammillary bodies [11].

Classical imaging findings in hippocampal sclerosis include volume loss and increased signal intensity in T2/ FLAIR images [12]. Increased signal intensity of hippocampus with atrophy visually was found in 13 (24.53\%) patients on the right side and $9(16.98 \%)$ in patients on the left side. Six out of 53 cases had bilateral hippocampal atrophy without corresponding increase in signal intensity in T2.

Atrophy is the most specific and reliable feature of hippocampal sclerosis (HS) [13].

The significance of extent of atrophy of hippocampus becomes important in assessing the prognosis after surgery for mesial temporal sclerosis. In a study by Kim y 


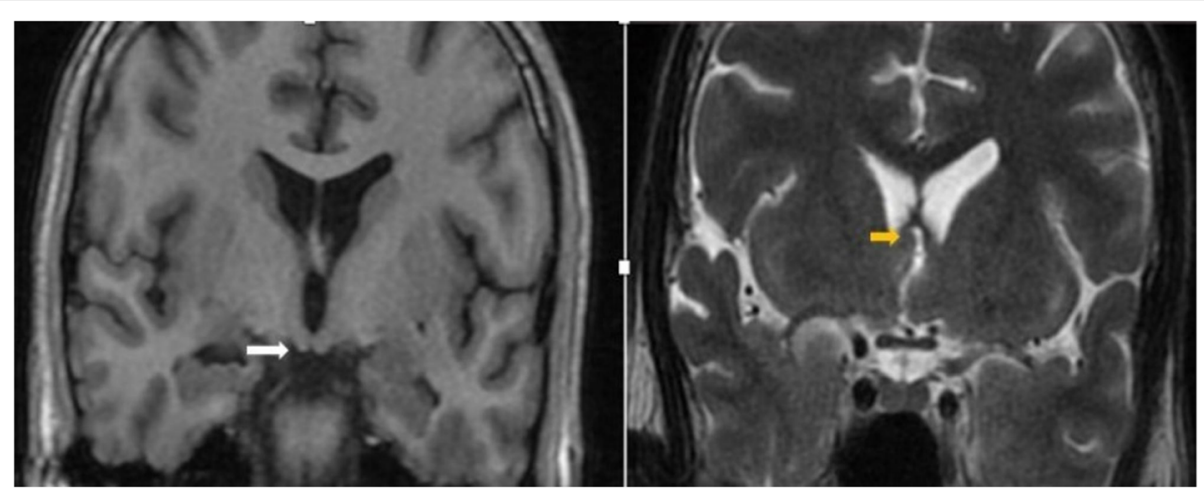

Fig. 3 Coronal MRI of brain showing mammillary body atrophy on the right side (white arrow) and right fornix atrophy (yellow arrow) in the patient with focal onset seizure with impaired awareness

and colleagues, it was seen that an MR imaging finding of hippocampal atrophy is the most useful sole prognostic indicator [13].

Visual identification of abnormal hippocampus is straightforward if one side is clearly normal and the other is abnormal. In symmetric bilateral disease or mild unilateral disease, visual analysis may produce problems [11].

The secondary findings in the mammillary bodies and fornix on MR imaging help in the diagnosis and lateralization of MTS [14]. In our study out of 53 cases, 13 cases showed atrophy of mammillary bodies/fornix with temporal horn dilation ipsilateral to the side of hippocampal atrophy.
In patients with subtle findings of unilateral MTS, the secondary imaging features may add to improve diagnostic confidence. Although the secondary MR imaging findings associated with MTS are not sensitive predictors of this entity by themselves, they may offer clues in subtle cases [15].

Quantitative hippocampal volumetry has been shown to predict postsurgical outcome in various studies; however, according to Kim y and colleagues, the interpretation of MR images by visual inspection alone has a similar prognostic value [12].

Quantitatively, 15 (28.30\%) subjects had hippocampal atrophy volumetrically vs. $13(24.53 \%)$ visually on the right side, whereas on the left side, only $9(16.98 \%)$

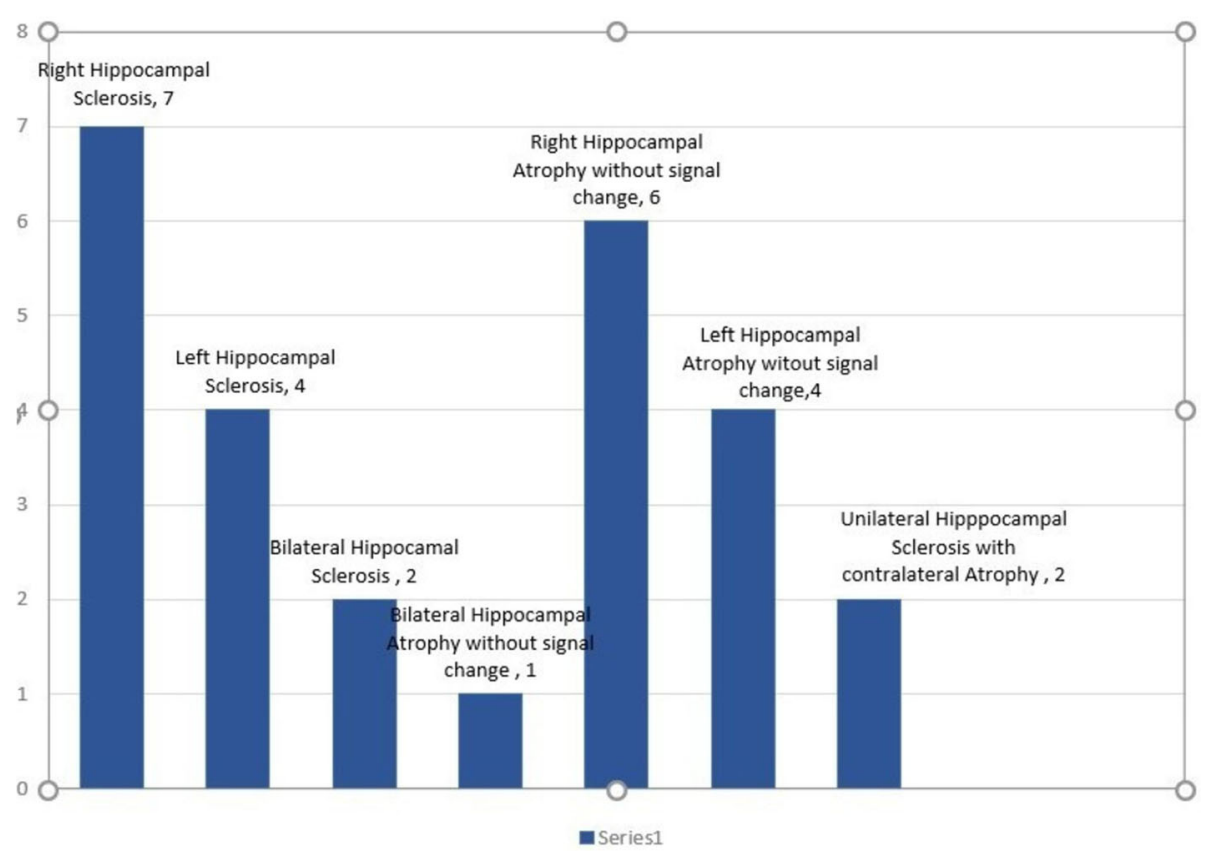

Fig. 4 Distribution of hippocampal atrophy with T2 hyperintensity in patients with focal onset seizure with impaired awareness 
Table 3 Mean T2 relaxometry of hippocampal sclerosis in comparison with normal hippocampus in patients with focal onset seizures with impaired awareness

\begin{tabular}{llll}
\hline Side & Normal & Hippocampal sclerosis & $\boldsymbol{p}$ value \\
\hline Right & 71.32 & 81.44 & $0.008(\mathrm{t}-2.482)$ \\
Left & 76.13 & 86 & $0.048(\mathrm{t}-1.725)$ \\
\hline
\end{tabular}

subjects were detected visually, while 10 (18.87\%) were detected volumetrically to have atrophy. In our study, by volumetry, we were able to detect more cases of atrophy quantitatively which was not evident on visual inspection alone.

Another important indicator of hippocampal sclerosis is increased hippocampal T2 signal which indicates gliosis [13]. The degree and extent of hippocampal gliosis also correlate with the T2 signal in the hippocampus.

MR imaging studies by Bronen RA, Jackson GD, and colleagues have described a variable frequency of T2 signal change in the hippocampus: change was observed in 12 to $65 \%$ of patients with hippocampal sclerosis [13].

But in our study, T2 hyperintensity were found in $30.18 \%$. In our study, there is a single case with T2 hyperintensity of hippocampus ipsilateral to the clinical and EEG localisation of the seizure focus (right sided), however with no atrophy.

As per study by Kim y and colleagues, it has been suggested that finding of hippocampal atrophy is more useful than one of high $\mathrm{T} 2$ signal in determining hippocampal sclerosis by histology. As per Kim and colleagues, hippocampal atrophy was much more common finding than high T2 signal (97\% vs. 61\%).

$\mathrm{T}_{2}$-weighted hyperintensity is one of the salient radiologic features of HS which can be objectively assessed by quantitative measurement of $\mathrm{T} 2$ relaxation (T2 mapping). It has got higher sensitivity over visual analysis. It is of great importance to note that $\mathrm{T} 2$ values may be elevated even in the absence of atrophy [16]. Thus, the combination of hippocampal atrophy with an elevated T2 value is both sensitive and specific for HS. As a result, combining findings of hippocampal volumes and T2 values can increase the yield to $99 \%$ of visually detected HS, but also $28 \%$ of those considered visually normal [1].

In our study, comparisons of the right and left hippocampal T2 relaxometry values in subjects with normal and abnormal hippocampus were found to have a statistically significant difference.

Jackson G. D and colleagues performed hippocampal T2 relaxometry as routine MRI examination and concluded that abnormal T2 relaxometry is significantly associated with intractable epilepsy [17].

\section{Conclusion}

Visual inspection alone may be sufficient to diagnose hippocampal sclerosis, but in cases with subtle volume loss or bilateral atrophy, volumetry serves as an expert eye. Secondary signs such as atrophy of mammillary body, fornix, and prominence of the temporal horn of the lateral ventricle are of paramount significance while assessing the hippocampal atrophy visually especially if the pathology is bilateral.

T2 relaxometry turns out to be a useful tool in detecting hippocampal sclerosis, as a striking difference is noted in cases with hippocampal sclerosis and those presumed to have normal hippocampus.

\section{Limitation}

1. MTS accounts for the majority of the cases of focal onset seizure with impaired awareness (CPS), and hence MRI is done to confirm the diagnosis as a pre-operative work-up. However, since surgery for hippocampal sclerosis is not performed in our institution, most of the patients suspected with hippocampal sclerosis are referred to centres where surgery is performed and evaluated with MRI there.

2. Limited sample size.

3. The results of T2 relaxometry is equipment specific, and therefore, variations may occur based on the equipment used for evaluation.

4. The study has not considered the frequency of seizure episodes in assessing the hippocampal atrophy.

\section{Abbreviations}

CPS: Complex partial seizures; ILAE: The International League Against Epilepsy; HS: Hippocampal sclerosis; MRI: Magnetic resonance imaging; MTS: Mesial temporal sclerosis; TLE: Temporal lobe epilepsy

\section{Acknowledgements}

We are grateful to the neurology department and the senior neurologist for referring the cases for MRI. We are grateful to the technologists/

radiographers for acquiring the MRI images according to seizure protocol.

\begin{abstract}
Authors' contributions
The corresponding author MA and first author AEA chose the idea and put the proposal. The first author AEA is the principal investigator, researched the literature and conceived the study, and was involved in protocol development, gaining of ethical approval, and data collection and analysis. The second author NP and third author JP were the two senior readers/ observers who analysed the MRI. The corresponding author MA helped the first author AEA in the drafting of the manuscript. All authors reviewed and edited the manuscript and approved the final version of the manuscript.
\end{abstract}

\section{Funding}

This research required no external funding from any agency. The entire expense was met by the principal investigator. 


\section{Declarations}

\section{Ethics approval and consent to participate}

This work has been accepted by the institutional research and ethics committee of Government Medical College, Kozhikode (Calicut), on 26 October 2017 (reference number GMCKKD/RP 2017/EC/176).

Consent to participate has been approved also by the same committee.

\section{Consent for publication}

Not applicable

\section{Competing interests}

The authors have no conflict of interests.

\section{Author details}

'Department of Radiodiagnosis, Government Medical College, Thrissur, Kerala, India. ${ }^{2}$ Department of Radiodiagnosis, Government Medical College Kozhikode, Kozhikode, India. ${ }^{3}$ Professor of Medicine, Government Medical College Thrissur, Thrissur, Kerala 680002, India. ${ }^{4}$ Mekkattukunnel House,

Chakkoth lane, Poonkunnam, Thrissur, Kerala 680002, India.

Received: 7 April 2021 Accepted: 25 June 2021

Published online: 23 July 2021

\section{References}

1. Singh P, Kaur R, Saggar K, Singh G, Kaur A. Qualitative and quantitative hippocampal MRI assessments in intractable epilepsy. Biomed Res Int. 2013; 2013:480524.

2. Kramer U, Riviello JJ Jr, Carmant L, Black PM, Madsen J, Holmes GL. Clinical characteristics of complex partial seizures: a temporal versus a frontal lobe onset. Seizure. 1997;6(1):57-61. https://doi.org/10.1016/S1059-1311 (97)80054-4

3. Vos SB, Winston GP, Goodkin O, Pemberton HG, Barkhof F, Prados F, et al. Hippocampal profiling: localized magnetic resonance imaging volumetry and T2 relaxometry for hippocampal sclerosis. Epilepsia. 2020;61 (2):297-309. https://doi.org/10.1111/epi.16416.

4. Farid N, Girard HM, Kemmotsu N, Smith ME, Magda SW, Lim WY, et al. Temporal lobe epilepsy: quantitative MR volumetry in detection of hippocampal atrophy. Radiology. 2012;264(2):542-50. https://doi.org/10.114 8/radiol.12112638

5. Mohandas AN, Bharath RD, Prathyusha PV, Gupta AK. Hippocampal volumetry: normative data in the Indian population. Ann Indian Acad Neurol. 2014;17(3):267-71. https://doi.org/10.4103/0972-2327.138482.

6. Fisher RS, Cross JH, D'Souza C, French JA, Haut SR, Higurashi N, et al. Instruction manual for the ILAE 2017 operational classification of seizure types. Epilepsia. 2017;58(4):531-42. https://doi.org/10.1111/epi.13671.

7. Owolabi L, Sale S, Owolabi S. Clinico-electroencephalography pattern and determinant of 2-year seizure control in patients with complex partial seizure disorder in Kano, Northwestern Nigeria. Ann Med Health Sci Res. 2014;4(2):186-91. https://doi.org/10.4103/2141-9248.129030.

8. Williamson PD, French JA, Thadani VM, Kim JH, Novelly RA, Spencer SS, et al. Characteristics of medial temporal lobe epilepsy: II. Interictal and ictal scalp electroencephalography, neuropsychological testing, neuroimaging, surgical results, and pathology. Ann Neurol. 1993;34(6):781-7. https://doi.org/10.1 002/ana.410340605.

9. Brooks BS, King DW, el Gammal T, Meador K, Yaghmai F, Gay JN, et al. MR imaging in patients with intractable complex partial epileptic seizures. AJNR Am J Neuroradiol. 1990:11(1):93-9.

10. Muhlhofer W, Tan YL, Mueller SG, Knowlton R. MRI-negative temporal lobe epilepsy-What do we know? Epilepsia. 2017;58(5):727-42. https://doi.org/1 0.1111/epi.13699.

11. Cendes F, Theodore WH, Brinkmann BH, Sulc V, Cascino GD. Neuroimaging of epilepsy. Handb Clin Neurol. 2016;136:985-1014. https://doi.org/10.1016/ B978-0-444-53486-6.00051-X.

12. Dou W, Zhao L, Su C, Lu Q, Liu Q, Guo J, et al. A quantitative MRI index for assessing the severity of hippocampal sclerosis in temporal lobe epilepsy. BMC Med Imaging. 2020;20(1):42. https://doi.org/10.1186/s12880-020-00440-z.

13. Kim YH, Chang KH, Park SW, Koh YW, Lee SH, Yu IK, et al. Hippocampal sclerosis: correlation of MR imaging findings with surgical outcome. Korean J Radiol. 2001;2(2):63-7. https://doi.org/10.3348/kjr.2001.2.2.63.
14. Bote RP, Blázquez-Llorca L, Fernández-Gil MA, Alonso-Nanclares L, Muñoz A, De Felipe J. Hippocampal sclerosis: histopathology substrate and magnetic resonance imaging. Semin Ultrasound CT MR. 2008;29(1):2-14. https://doi. org/10.1053/j.sult.2007.11.005

15. Kuzniecky R, de la Sayette V, Ethier R, Melanson D, Andermann F, Berkovic S, et al. Magnetic resonance imaging in temporal lobe epilepsy: pathological correlations. Ann Neurol. 1987;22(3):341-7. https://doi.org/10.1002/ana.41022 0310.

16. Winston GP, Vos SB, Burdett JL, Cardoso MJ, Ourselin S, Duncan JS. Automated T2 relaxometry of the hippocampus for temporal lobe epilepsy. Epilepsia. 2017:58(9):1645-52. https://doi.org/10.1111/epi.13843.

17. Jackson GD, Connelly A, Duncan JS, Grünewald RA, Gadian DG. Detection of hippocampal pathology in intractable partial epilepsy: increased sensitivity with quantitative magnetic resonance T2 relaxometry. Neurology. 1993; 43(9):1793-9. https://doi.org/10.1212/WNL.43.9.1793.

\section{Publisher's Note}

Springer Nature remains neutral with regard to jurisdictional claims in published maps and institutional affiliations.

\section{Submit your manuscript to a SpringerOpen ${ }^{\circ}$ journal and benefit from:}

- Convenient online submission

- Rigorous peer review

- Open access: articles freely available online

- High visibility within the field

- Retaining the copyright to your article

Submit your next manuscript at $\boldsymbol{\nabla}$ springeropen.com 